\title{
KRT13, FAIM2 and CYP2W1 mRNA Expression in Oral Squamous Cell Carcinoma Patients with Risk Habits
}

\author{
Firstine Kelsi Hartanto ${ }^{1}$, Lee Peng Karen-Ng², Vui King Vincent-Chong², Siti \\ Mazlipah Ismail ${ }^{1}$, Wan Mahadzir Wan Mustafa ${ }^{3}$, Mannil Thomas Abraham³, \\ Keng Kiong Tay ${ }^{3}$, Rosnah Binti Zain ${ }^{1,2 *}$
}

\begin{abstract}
Background: Expression of KRT13, FAIM2 and CYP2W1 appears to be influenced by risk habits, thus exploring the associations of these genes in oral squamous cell cancer (OSCC) with risk habits, clinico-pathological parameters and patient survival may be beneficial in identifying relevant biomarkers with different oncogenic pathways. Materials and Methods: cDNAs from 41 OSCC samples with and without risk habits were included in this study. Quantitative real-time PCR was used to analyze KRT13, FAIM2 and CYP2W1 in OSCC. The housekeeping gene (GAPDH) was used as an endogenous control. Results: Of the 41 OSCC samples, KRT13 was down-regulated in 40 samples $(97.6 \%)$, while FAIM2 and CYP2W1 were down-regulated in $61.0 \%$ and $48.8 \%$, respectively. Overall, there were no associations between KRT13, FAIM2 and CYP2W1 expression with risk habits, selected socio-demographic and clinico-pathological parameters and patient survival. Conclusions: Although this study was unable to show significance, there were some tendencies in the associations of KRT13, FAIM2 and CYP2W1 expression in OSCC with selected clinic-pathological parameters and survival.
\end{abstract}

Keywords: CYPW1 - FAIM2 - KRT13 - oral squamous cell carcinoma - risk habits

Asian Pac J Cancer Prev, 16 (3), 953-958

\section{Introduction}

Oral cancer which ranked sixth together with pharyngeal cancer was among the most common malignancy of the head and neck region. Despite the advancement of treatment modalities over the last decades, about $50 \%$ of the patients afflicted still succumb to this debilitating disease within 5-year of diagnosis (Warnakulasurya, 2009). Majority of the oral cancer in the head and neck region is oral squamous cell carcinoma (OSCC) (Scully and Bagan, 2009). According to Ferlay et al. (2008), the annual incidence estimation is 263 cases per 100,000 for both male and female, while the overall mortality is $127(1.70 \%)$ per 100,000 .

The risk factors of OSCC are multifactorial, including genetics (e.g. carcinogen metabolism, defect in DNA repair and immunity), environmental (e.g. chemical and physical), lifestyle (e.g. habits of tobacco smoking, alcohol consumption, betel-quid chewing and diet) and microorganism (e.g. HPV16) (Scully and Bagan, 2009; Loyha et al., 2012; Krishna et al., 2013; Gupta and Johnson, 2014). Globally, 25\% of OSCC development may be due to tobacco use (smoking and/or chewing),
$7-19 \%$ to alcohol drinking, $10-15 \%$ to micronutrient deficiency and more than $50 \%$ to betel quid chewing in high chewing prevalence areas (Petti, 2009).

A few microarray-based gene-expression analyses that have been conducted found multiple genes regulated in cancer tissue as compared to normal tissue (Fialka et al., 2008) and some is associated with risk habits (Cheong et al., 2009). A recent preliminary study found these genes, KRT13, FAIM2 and CYP2W1 to be differentially expressed and down-regulated in OSCC as compared to normal samples. Expressions of these genes seem to be influenced by risk habits, thus exploring the associations of these genes in OSCC with and without risk habits are desirable and beneficial in identifying relevant biomarkers or predictive markers in OSCC with risk habits. Hence, the purpose of this study were to determine gene expressions particularly KRT13, FAIM2 and CYP2W1 that may be related to OSCC patients with and without risk habits, and also to investigate the associations between these genes expression with selected socio-demographic and clinic-pathological parameters. While KRT13 was well studied in OSCC, this is the first study involving FAIM2 and CYP2W1 expressions in OSCC.

${ }^{1}$ Department of Oral-Maxillofacial Surgical and Medical Sciences, ${ }^{2}$ Oral Cancer Research \& Coordinating Centre, Faculty of Dentistry, University of Malaya, Kuala Lumpur, ${ }^{3}$ Oral Health Division, Ministry of Health Malaysia, Putrajaya, Malaysia *For correspondence:rosnahmz@um.edu.my 


\section{Materials and Methods}

Samples

This is a cross-sectional study looking into the expression of genes KRT13, CYP2W1 and FAIM2 in OSCC using synthesized cDNA from fresh frozen tissue of patients with risk habits. A total of 44 (forty-four) cDNA synthesized from total RNA of fresh frozen tissues were included in this study, consisting of 41 tumour (OSCC) tissues as test samples and three alveolar mucosal tissues from non-cancer patient as reference for normalization of the test samples. Our study group consisted of cases of primary OSCC from patients who have not been treated and have complete socio-demographic and clinicopathologic data. Meanwhile, the non-cancer tissues were taken from alveolar mucosal tissue of individuals during the removal of impacted 8 in the minor oral surgery who do not have OSCC and other cancers, or potentially malignant disorders, and do not have risk habits. All cDNA, socio-demographic and clinico-pathological data were obtained from the Malaysian Oral Cancer Data and Tissue Bank System (MOCDTBS) at Oral Cancer Research and Coordinating Centre, University Of Malaya (OCRCC-UM). Details of the socio-demographic and clinico-pathological data were summarized in Table 1 . This study was approved by the Medical Ethics Committee at the Faculty of Dentistry, University of Malaya [MEC number: DF OP1202/0003(P)].

Table 1.Summary of Socio-Demographic and ClinicoPathological Parameters for 41 OSCC Samples Included in this Study

\begin{tabular}{|c|c|c|}
\hline Characteristics & $\mathrm{n}$ & Percentage $(\%)$ \\
\hline \multicolumn{3}{|l|}{ Gender } \\
\hline Male & 14 & 34.1 \\
\hline Female & 27 & 65.9 \\
\hline \multicolumn{3}{|l|}{ Age } \\
\hline$<40$ & 5 & 12.2 \\
\hline$>40$ & 36 & 87.8 \\
\hline \multicolumn{3}{|l|}{ Ethnicity } \\
\hline Malay & 11 & 26.2 \\
\hline Chinese & 8 & 19.5 \\
\hline Indian & 20 & 48.8 \\
\hline Iban & 1 & 2.4 \\
\hline Bidayuh & 1 & 2.4 \\
\hline \multicolumn{3}{|l|}{ Site } \\
\hline Tongue & 16 & 39 \\
\hline Gingiva & 8 & 19.5 \\
\hline Floor of mouth & 1 & 2.4 \\
\hline Buccal mucosa & 16 & 39 \\
\hline \multicolumn{3}{|l|}{ Habits } \\
\hline No habits & 13 & 31.7 \\
\hline Smoking & 9 & 22 \\
\hline Betel-quid chewing & 19 & 46.3 \\
\hline \multicolumn{3}{|l|}{ Tumour differentiation* } \\
\hline Well-differentiated & 20 & 48.8 \\
\hline Moderately-differentiated & 20 & 48.8 \\
\hline \multicolumn{3}{|l|}{ Tumour staging } \\
\hline Early (I \& II) & 12 & 29.3 \\
\hline Advance (III \& IV) & 29 & 70.7 \\
\hline \multicolumn{3}{|l|}{ Lymph node metastasis } \\
\hline No & 24 & 58.5 \\
\hline Yes & 17 & 41.5 \\
\hline
\end{tabular}

Quantitative PCR ( $q P C R$ ) analysis

$2 \mathrm{ng} / \mu \mathrm{l} \mathrm{cDNA}$ of OSCC and normal tissues were utilized in this analysis. qPCR was performed on a 7500 Fast Real-Time PCR System (Applied Biosystems, Foster City, CA, USA) in triplicates on the cDNA samples using TaqMan Fast Universal PCR master mixture (Applied Biosystems, Foster City, CA, USA). The TaqMan Gene Expression Assays (Applied Biosystems, Foster City, CA, USA) used were; KRT13 (Hs00357961_gl), CYP2W1 (Hs00214994_ml) and FAIM2 (Hs00202349_ml). All qPCR reactions were carried out according to the manufacturer's protocol (Applied Biosystems, Foster City, CA, USA). Relative quantification/fold change (RQ) of all genes was calculated using $2^{-\Delta \Delta C T}$ method in 7500 Fast System SDS Software 2.0.5 (Applied Biosystems, Foster City, CA, USA). The housekeeping gene (GAPDH) was used as endogenous control while the cDNA from normal oral mucosa tissue was utilized to normalize test samples (OSCC).

\section{Statistical analysis}

The expressions of the genes can be categorized into 3 groups, which are down-regulated $(\mathrm{RQ} \leq 0.50)$, unchanged $(0.51<R Q<1.99)$ and up-regulated $(R Q \geq 2)$. Mann-Whitney (comparing two groups) and KruskalWallis (comparing more than 2 groups) test were used to investigate the association between KRT13, FAIM and CYP2W1 expressions with risk habits (smoking and betel-quid chewing), selected socio-demographic data (age, gender and ethnicity) and clinico-pathologic feature (lymph node metastasis). Kaplan-Meier test was carried out to determine the association of KRT13, FAIM2, and CYP2W1 expressions with survival of oral cancer patients with risk habits. P-values $<0.05$ was considered to be statistically significant. All the tests were performed by SPSS 12.01 for Windows.

\section{Results}

Expressions of KRT13, FAIM2 and CYP2W1

Overall, KRT13, FAIM2 and CYP2W1 were expressed in all 41 OSCC samples. KRT13 showed significant down-regulation expression (fold change $>10$ ) in OSCC after normalization with non-OSCC samples. FAIM2 and CYP2W1 showed relatively unchanged expressions with fold change of- 1.70 and- 0.83 respectively. Expressions of these genes were illustrated in Figure 1.

Of the 41 OSCC samples, KRT13 was down-regulated in 40 samples (97.6\%). However, expression of FAIM2 showed down-regulation in 25/41 of the samples (61.0\%), unchanged expression in 10/41 (24.4\%) samples and upregulated in 6/41 (14.6\%) samples. Meanwhile, CYP2W1 was found to be down-regulated in 20/41 (48.8\%) samples, followed by up-regulated expression in 13/41 (31.7\%) and unchanged in 8/41 (19.5\%) samples.

Association between KRT13, FAIM2 and CYP2W1 expressions with risk habits

Mann-Whitney and Kruskal-Wallis tests were performed to investigate the association between each gene expression with risk habits (smoking and betel-quid 
chewing). Overall result showed that KRT13, FAIM2 and CYP2W1 expressions were not associated with the risk habits $(\mathrm{p}>0.05)$. However, while down-regulation of CYP2W1 was seen among chewers and no habits group, there seems to be a trend among the smokers that the expression of CYP2W1 was found to be up-regulated although it was not statistically significant $(\mathrm{p}=0.34)$. Table 2 summarized the association between the expressions of KRT13, FAIM2 and CYP2W1 with risk habits.

Association between KRT13, FAIM2 and CYP2W1 expressions with selected socio-demographic and clinicopathological parameters

Mann-Whitney and Kruskal-Wallis tests were also employed to investigate the association between KRT13, FAIM2 and CYP2W1 expressions with selected sociodemographic and clinico-pathological parameters (age, gender, ethnicity and lymph node metastasis). In general, there were no significant difference between KRT13, FAIM2 and CYP2W1 expressions with age, gender, ethnicity and lymph node metastasis. The expressions of KRT13 and FAIM2 showed down-regulation in all the parameters studied. However, among the Chinese, there seems to be an up-regulation of CYP2W1 expression although it was not statistically significant $(p=0.29)$. The association between the expressions of KRT13, FAIM2 and CYP2W1 and selected socio-demographic and clinicpathological parameter were summarized in Table 3 .

Association between KRT13, FAIM2 and CYP2W1 expressions with patients' survival

Kaplan Meier analysis was performed to investigate the association between expressions of KRT13, FAIM2 and CYP2W1 with overall patients' survival. The expression of KRT13 was excluded from the analysis because 40/41 samples were found to be down-regulated. Overall, there were no significant associations between FAIM2 ( $\mathrm{p}=0.48)$ and CYP2W1 $(\mathrm{p}=0.19)$ expressions with patients' survival.

At the $30^{\text {th }}$ month, there was a $60 \%$ survival rate among OSCC patients with down-regulation of FAIM2 as compared to about $90 \%$ overall survival among those with up-regulation of this gene, although it was not statistically significant (Figure 2). Meanwhile up-regulation of CYP2W1 expression seems to have better overall patients' survival $(75 \%)$ as compared to down-regulation of this

Table 2. Association between KRT13, FAIM2 and CYP2W1 Expressions with Risk Habits

\begin{tabular}{|c|c|c|c|c|c|c|c|}
\hline \multirow{2}{*}{$\begin{array}{l}\text { The risk habits and } \\
\text { genes expressions }\end{array}$} & \multirow{2}{*}{$\frac{n}{41}$} & \multicolumn{2}{|c|}{ KRT13 } & \multicolumn{2}{|c|}{ FAIM2 } & \multicolumn{2}{|c|}{ CYP2W1 } \\
\hline & & Median (IQR) & P-value & Median (IQR) & P-value & Median (IQR) & $P$ P-value \\
\hline \multicolumn{8}{|l|}{ Habits } \\
\hline No habits & 13 & $0.00(0.02)$ & $0.86^{\mathrm{a}}$ & $0.24(0.45)$ & $0.16^{\mathrm{a}}$ & $0.61(2.65)$ & $0.34^{\mathrm{a}}$ \\
\hline Smoking & 9 & $0.00(0.04)$ & & $0.36(0.66)$ & & $2.55(19.9)$ & \\
\hline Betel-quid chewing & 19 & $0.00(0.04)$ & & $0.87(1.21)$ & & $0.39(1.48)$ & \\
\hline \multicolumn{8}{|l|}{ Habits } \\
\hline No habits & 13 & $0.00(0.02)$ & $0.81^{\mathrm{b}}$ & $0.24(0.45)$ & $0.10^{\mathrm{b}}$ & $0.61(2.57)$ & $0.97^{\mathrm{b}}$ \\
\hline With habits & 38 & $0.02(0.03)$ & & $0.45(0.82)$ & & $0.44(3.31)$ & \\
\hline \multicolumn{8}{|l|}{ Habits * } \\
\hline No habits & 13 & $0.00(0.02)$ & $0.65^{\mathrm{b}}$ & $0.24(0.45)$ & $0.37^{\mathrm{b}}$ & $0.61(2.57)$ & $0.41^{\mathrm{b}}$ \\
\hline Currently smoke & 8 & $0.00(0.01)$ & & $0.42(0.71)$ & & $1.64(27.8)$ & \\
\hline \multicolumn{8}{|l|}{ Habits $* *$} \\
\hline No habits & 13 & $0.00(0.02)$ & $0.59^{b}$ & $0.24(0.45)$ & $0.11^{\mathrm{b}}$ & $0.61(2.57)$ & $0.44^{\mathrm{b}}$ \\
\hline Currently chew & 18 & $0.00(0.05)$ & & $0.88(1.62)$ & & $0.35(0.91)$ & \\
\hline
\end{tabular}

*Samples with betel-quid chewing habit were excluded; **Samples with smoking habit were excluded; a Kruskal Wallis test was performed, level of significance is 0.05 b Mann Whitney test was performed, level of significance is 0.05

Table 3. Association between KRT13, FAIM2 and CYP2W1 Expressions with Selected Socio-Demographic and Clinico-Pathological Parameters in 41 Samples

\begin{tabular}{|c|c|c|c|c|c|c|c|}
\hline \multirow{2}{*}{$\begin{array}{l}\text { Socio-demographic and } \\
\text { clinico-pathological } \\
\text { parameters }\end{array}$} & \multicolumn{3}{|c|}{ KRT13 } & \multicolumn{2}{|c|}{ FAIM2 } & \multicolumn{2}{|c|}{ CYP2W1 } \\
\hline & n (41) & Median (IQR) & P-value & Median (IQR) & P-value & Median (IQR) & $\mathrm{P}$-value \\
\hline \multicolumn{8}{|l|}{ Gender } \\
\hline Male & 14 & $0.00(0.02)$ & $0.51^{\mathrm{a}}$ & $0.28(0.71)$ & $0.28^{\mathrm{a}}$ & $0.61(2.94)$ & $0.99^{\mathrm{a}}$ \\
\hline Female & 27 & $0.00(0.04)$ & & $0.41(0.91)$ & & $0.45(2.96)$ & \\
\hline \multicolumn{8}{|l|}{ Age } \\
\hline$<40$ & 5 & $0.00(0.10)$ & $0.14^{\mathrm{a}}$ & $0.25(0.54)$ & $0.32^{\mathrm{a}}$ & $0.23(2.13)$ & $0.27^{\mathrm{a}}$ \\
\hline$>40$ & 36 & $0.00(0.04)$ & & $0.37(0.88)$ & & $0.52(3.00)$ & \\
\hline \multicolumn{8}{|l|}{ Ethnicity * } \\
\hline Malay & 11 & $0.00(0.00)$ & $0.49^{\mathrm{b}}$ & $0.25(0.66)$ & $0.16^{\mathrm{b}}$ & $0.72(4.06)$ & $0.29^{b}$ \\
\hline Chinese & 8 & $0.02(0.17)$ & & $0.26(0.42)$ & & $1.33(2.37)$ & \\
\hline Indian & 20 & $0.00(0.04)$ & & $0.88(1.64)$ & & $0.41(2.81)$ & \\
\hline \multicolumn{8}{|l|}{ Lymph node metastasis } \\
\hline No & 24 & $0.00(0.02)$ & $0.97^{\mathrm{a}}$ & $0.33(0.78)$ & $0.85^{\mathrm{a}}$ & $0.49(3.15)$ & $0.30^{\mathrm{a}}$ \\
\hline Yes & 17 & $0.00(0.07)$ & & $0.49(1.84)$ & & $0.50(2.39)$ & \\
\hline
\end{tabular}

*In comparison of genes expressions in different ethnicity, two samples (one each of Iban and Bidayuh ethnicity) were excluded in the statistical analysis due to its small number; a Mann Whitney test was performed, level of significance is 0.05 ; b Kruskal Wallis test was performed, level of significance is 0.05 
Genes expressions in OSCC

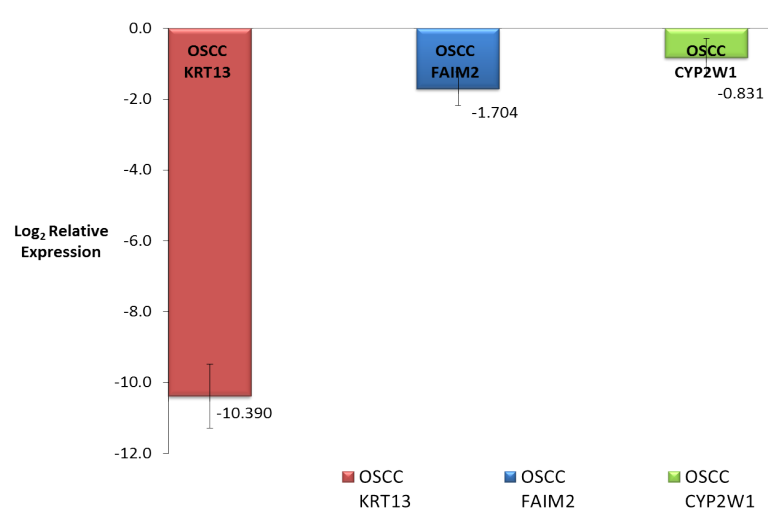

Figure 1.Expressions of KRT13,FAIM2, and CYP2W1 in 41 OSCC samples. KRT13 showed significantly downregulated, while FAIM2 and CYP2W1 were relatively unchanged

Survival Functions

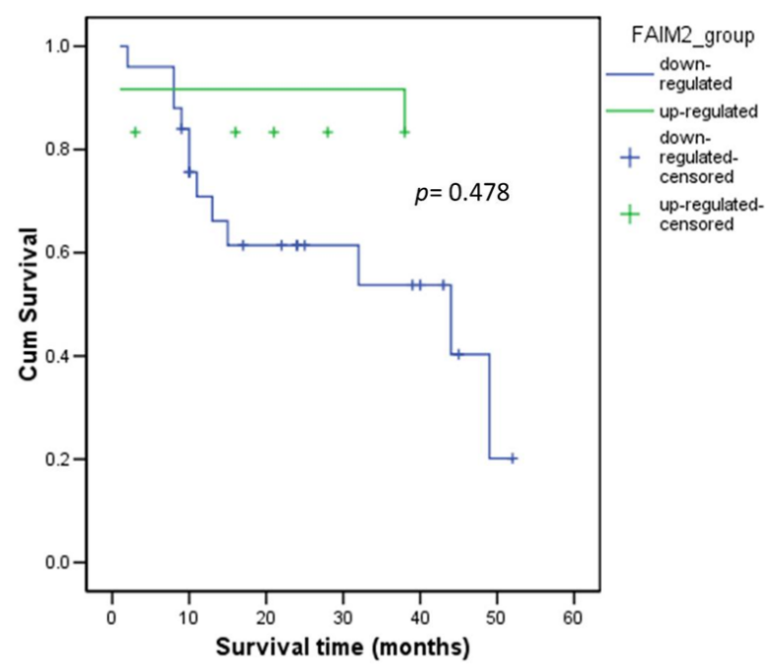

Figure 2. Survival of OSCC Patients in Relation with FAIM2 Expression

Survival Functions

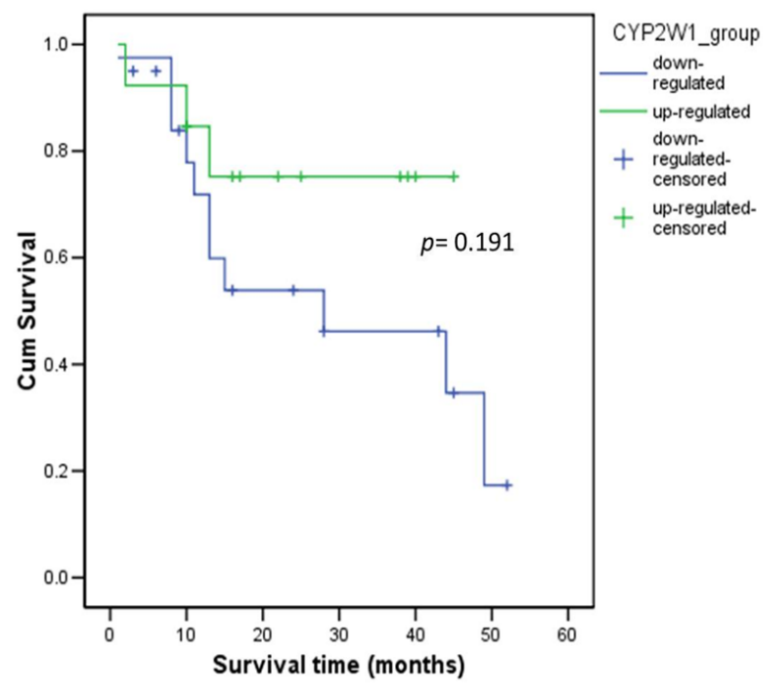

Figure 3. Survival of OSCC Patients in Relation with CYP2W1 Expression gene with $45 \%$ at month 30 , again, it was not statistically significant $(\mathrm{p}=0.19)$ (Figure 3 ).

\section{Discussion}

The keratins are intermediate filament protein responsible for structural integrity of epithelial cells. KRT13 is a member of keratin family, which plays role in cytoskeleton remodeling. It is one of the genes that currently found to be dysregulated in any location of tumour. In the present study, KRT13 showed significantly down-regulated in OSCC as compared with non-OSCC. This finding was in concordance with a study by Lallemant et al. (2009) using qPCR assay which concurred that KRT13 expression was found lower in the head and neck cancer tumour tissues as compared with normal tissues. In the immunohistochemical studies, KRT13 was $100 \%$ expressed in normal mucosa, mild and moderate oral epithelial dysplasia. The expression was observed throughout the epithelial layer except for basal cells. In contrast, KRT13 was absent in carcinoma in situ and OSCC. It was suggested that the expression of KRT13 might has a link with epithelial differentiation (Bloor et al., 2001; Mikami et al., 2011). The loss expression of KRT13 expressions had also been reported in cervix carcinoma tissue as the normal tissue progress to become a neoplastic cell (Carrilho et al., 2004). KRT13 can be used as marker of malignant transformation and differentiation of stratified squamous epithelium.

Apoptosis or programmed cell death played few roles in biological phenomena, such as modeling the embryo, regulating the immune system and tumour regression. However, dysfunction of this system has been implicated in oncogenesis, development in degenerative disease and autoimmunity. FAIM2 (Fas apoptotic inhibitory molecule 2), also known as LFG (lifeguard) is one of the genes that played the role in protecting cells from Fas-induced apoptosis pathway, but not from TNF- $\alpha$ pathway. To the best of our knowledge, this is the first study about FAIM2 expression in OSCC. In the present study, FAIM2 found to be down-regulated in OSCC. The high expression of LFG was found in the hippocampus and mostly in the neuron cells (Somia et al., 1999). Reich et al. (2011) found that loss of FAIM2 will result in enhanced vulnerability to cerebral ischemia with increased neuronal cell death, ischemic lesion volume, and neurological impairment. It was speculated that the expression of FAIM2 in OSCC was associated with survival of the tumour cells, regardless upregulated or down-regulated. FAIM2 is also one of genes that associated with obesity. Obesity is a risk factor for type II diabetes, artheroclerosis, and some forms of cancer (Boender et al., 2012). Obesity (mainly due to excessive food intake) together with tobacco smoking and alcohol drinking are part of unhealthy lifestyle which could lead to development of cancer in later years. However, the mechanisms of FAIM2 in these events still remain unclear. In addition, the role of FAIM2 in oral cancer associated with risk habits is yet to be elucidated.

CYP2W1 (cytochrome P450, family 2, polypeptide 1 ) is a new member of P450 family and is distributed at HepG2 cell in the form of mRNA and protein (Karlgren 
et al., 2005). This gene plays role in biological process, oxidation-reduction process, small-molecule metabolic process, and xenobiotic metabolic process. In this study, CYP2W1 found to be down-regulated in majority OSCC $(48.8 \%)$ patients with risk habits. Only 10/41 (24.4\%) OSCC samples showed up-regulated of CYP2W1 expression. Meanwhile, other studies showed CYP2W1 over-expression in gastric cancer $(50.0 \%)$, colon cancer (78.0\%) and adrenal tumour (75.0\%) compared to normal tissue (Aung et al., 2006; Karlgren et al., 2006). An immunohistochemical study in colorectal cancer showed that CYP2W1 expression was associated with tumour staging and patients with higher CYP2W1 expression had worse clinical outcome in 5 and 10 year survival (Edler et al., 2009; Stenstedt et al., 2012). While most of the samples in this study showed down-regulation of CYP2W1, it could probably be due to different type of tumour and heterogeneity of the tumour itself.

In association with risk habits, particularly smoking and betel nut chewing, no significant association was found between KRT13, FAIM2 and CYP2W1 expressions with any of the risk habits (smoking, chewing and no habits). It was observed that the expressions of KRT13 and FAIM2 were down-regulated among smokers, chewers and no habits group. In particular, CYP2W1 expression seemed to be up-regulated in OSCC with smoking habit as compared to betel-quid chewing habit or without risk habits although it was not statistically significant. CYP2W1 has also been shown to participate in bioactivation of several pro-carcinogen including polycyclic and aflatoxin B1 (Nishida et al., 2011). It is possible that high expression of CYP2W1 in OSCC with smoking habit may be related to the action of this gene in the activation of pro-carcinogen contained in tobacco smoking such as aromatic hydrocarbons.

In the present study, there were no significant associations of KRT13, FAIM2 and CYP2W1 expressions with the selected socio-demographic parameters (gender, age and ethnicity) and lymph node metastasis. Overall, the expressions of KRT13, FAIM2 and CYP2W1 were found to be down-regulated among the socio-demographic and clinico-pathologic parameters (gender, age group, ethnicity and lymph node metastasis). Despite that there were no significant difference between any of the gene expressions (KRT13, FAIM2 and CYP2W1) with lymph node metastasis, the selection of this clinico-pathologic parameter in this study was vital as lymph node metastasis has become an important prognostic factor in predicting clinical outcome of cancer. It has also been accepted that lymph nodes metastasis is the main factor influencing survival in oral and pharyngeal squamous cell carcinoma (Woolgar, 1995). The present study also showed that CYP2W1 expression was up-regulated in Chinese ethnicity compared with Malays and Indians. However, no specific explanation could be given for this finding except for the fact that the heterogeneity of the tumours in different socio demographic situations.

In this study, no significant association was found between FAIM2 and CYP2W1 expressions with overall patients' survival. Comparatively at 30 months after first diagnosis, OSCC patients' overall survival seems to be better at $90 \%$ and $75 \%$ among those with up-regulation of FAIM2 and CYP2W1 expressions respectively. While the reason for these may be unknown, the finding by Edler et al. (2009) seems to be in contrast with the current study. In his study, he found that $64 \%$ of colorectal cancer expressed low level of CYP2W1 expression and 36\% expressed high level of CYP2W1expression and they concluded that its expression can be used as independent prognostic factor for survival, where a high expression was associated with a worse clinical outcome. It is possible that the difference in sample size in the study $(n=162)$ by Edler et al. (2009) from the current study $(n=41)$ as one of the factors influencing the current study result.

Regarding possible limitations, the present study only investigated a small sample size of 41 OSCC samples that were available. Within this 41 OSCC samples, several associations were tried to be established between the gene expressions with risk habits, socio-demographic, clinicpathological parameters and patients' survival. Upon dividing these samples into different subgroups according to risk habits, socio-demographic and clinico-pathologic parameters, the number of samples was smaller which raised concerns that it may not be representative to achieve significant findings. Some meaningful analysis such as to further compare association between gene expressions with current and former smokers within the smoking habit was also limited with small sample size.

In conclusion, the expressions of KRT13, FAIM2 and CYP2W1 were not influenced by any risk habits. KRT13, FAIM2 and CYP2W1 expressions were not associated with various socio-demographic parameters (gender, age, and ethnicity) and lymph node metastasis. In addition, KRT13, FAIM2 and CYP2W1 expressions also were not associated with overall patients' survival.

\section{Acknowledgements}

This research is supported by High Impact Research MoE Grant UM.C/625/1/HIR/MoE/Dent/08, University of Malaya Research Grant (RP002C-13HTM) and postgraduate research grant PPPC/C1-2012/DEGC/15.

\section{References}

Aung P.P, Oue N, Mitani Y, et al (2006). Systematic research for gastric cancer-specific genes based on SAGE data: melanoma inhibitory and matrixmetalloproteinase- 10 are novel prognostic factors in patients with gastric cancer. Oncogene, 25, 2546-57

Bloor BK, Seddon SV, Morgan PR (2001). Gene expression of differentiation-specific keratin in oral epithelial dysplasia and squamous cell carcinoma. Oral Oncol, 37, 251-61

Boender AJ, Rozen AJ, Adan RAH (2012). Nutritional state affects the expression of the obesity-associated genes Etv5, Faim2, Fto, and Negr1. Obesity, 20, 2420-5

Carrilho C, Alberto M, Buane L, et al (2004). Keratin 8, 10, 13, and 17 are useful markers in the diagnosis of human cervix carcinomas. Human Pathol, 35, 546-51

Cheong SC, Chadramouli GVR, Saleh A, et al (2009). Gene expression in human oral squamous cell carcinoma is influenced by risk factor exposure. Oral Oncol, 45, 712-9

Edler D, Stenstedt K, Ohrling K, et al (2009). The expression 
of the novel CYP2W1 enzyme is an independent prognostic factor in colorectal cancer - A pilot study. Eur J Cancer, 45, 705-12

Ferlay J, Shin HR, Bray F, et al (2010). Estimates of worldwide burden of cancer: GLOBOCAN 2008. Intern J Cancer, 127, 2893-917

Fialka F, Gruber RM, Hitt R, et al (2008). CPA6, FMO2, LGI1, SIAT1 and TNC are differentially expressed in early- and late-stage oral squamous cell carcinoma--a pilot study. Oral Oncol, 44, 941-8

Gomez A, Karlgren M, Edler D, et al (2007). Expression of CYP2W1 in colon tumour: regulation by gene methylation. Pharmacogenomics, 8, 1315-25

Gupta B, Johnson NW (2014). Emerging and established global life-style risk factors for cancer of the upper aero-digestive tract. Asian Pac J Cancer Prev, 15, 5983-91.

Karlgren M, Miura S, Ingelman-Sunberg M (2005). Novel extrahepatic cytochrome P450s. Toxicol Applied Pharmacol, 207, 57-61

Karlgren M, Gomez A, Stark K, et al (2006). Tumor-specific expression of the novel cytochrome P450 enzyme, CYP2W1. Biochem Biophys Res Commun, 341, 451-8

Krishna Rao SV, Mejia G, Roberts-Thomson K, et al (2013). Epidemiology of oral cancer in Asia in the past decade--an update (2000-2012). Asian Pac J Cancer Prev, 14, 5567-77

Lallemant B, Evrard A, Combescure C, et al (2009). Clinical relevance of nine transcriptional molecular markers for the diagnosis of head and neck squamous cell carcinoma in tissue and saliva rinse. BMC Cancer, 9, 370

Loyha K, Vatanasapt P, Promthet S, et al (2012). Risk factors for oral cancer in northeast Thailand. Asian Pac J Cancer Prev, 13, 5087-90

Mikami T, Cheng J, Maruyama S, et al (2011). Emergence of keratin 17 vs. loss of keratin 13: Their reciprocal immunohistochemical profiles in oral carcinoma in situ. Oral Oncol, 47, 497-503

Nishida CR, Lee M, De Montellano PR (2010). Efficient hypoxic activation of the anticanceragent AQ4N by CYPS1 and CYP2W1. Mol Pharmacol, 78, 497-502

Petti S (2009). Lifestyle risk factors for oral cancer. Oral Oncol, 45, 340-50

Reich A, Spering C, Gertz K, et al (2011). Fas/CD95 Regulatory Protein Faim2 is neuroprotective after transient Brain Ischemia. J Neurosci, 31, 225-33

Scully C, Bagan JV (2009). Oral squamous cell carcinoma: overview of current understanding of aetiopathogenesis and clinical implications. Oral Diseases, 15, 388-99

Somia NV, Schmitt MJ, Vetter DE, et al (1999). LFG: An antiapoptotic gene that provides protection from Fas-mediated cell death. Proc Nati Acad Sci U S A, 96, 12667-72

Stenstedt K, Hallstorm M, Johansson I, et al (2012). The expression of CYP2W1: a prognostic marker in colon cancer. Anticancer Res, 32, 3869-74

Warnakulasuriya S (2009). Global epidemiology of oral and oropharyngeal cancer. Oral Oncol, 45, 309-16

Woolgar J.A, Scott J, Vaughan E.D, et al (1995). Survival, metastasis and recurrence of oral cancer in relation to pathological features. Ann R Coll Surg Engl, 77, 325-31 